\title{
In ISM Modeling, The Devil is in the Details: You Show Me Your OVI and I'll Show You Mine.
}

Donald P. Cox

Department of Physics, University of Wisconsin-Madison, 1150 University Ave., Madison, WI 53706, USA, Email: cox@wisp.physics.wisc.edu

\begin{abstract}
The three sections of this paper illustrate the importance of rationalizing ISM theory and modeling with the observational information.
\end{abstract}

Keywords: ISM: general, ISM: Local Bubble, X-rays: galaxies

\section{Modeling the ISM is Complicated.}

On this topic, see also Maarit Korpi's contribution and two other papers of mine ("Guide to Modeling the Interstellar Medium," and "Overall Models of the Interstellar Medium," 1996a,b).

Imagine pouring the ISM into the Galaxy's gravitational potential, where it finds all the other constituents already in place. It is globally confined by gravity, stabilized radially by rotation, heated by starlight, disrupted by catastrophic stellar events, settles into components spanning a wide range of density and temperature, develops a magnetic field, confines cosmic rays, generates new stars, interacts with stellar density waves, and is concentrated by that into the gaseous spiral arms. It develops a vertical quasi-hydrostatics, with dynamic pressure, magnetic pressure (and tension), and cosmic ray pressure as dominant forms of support. When locally heated, it develops bubbles of hot gas that grow until their thermal pressures are in approximate equilibrium with the magnetic pressure that surrounds them. This can occur either via random heating events (accidental sequences of supernovae in proximity), or via the local production of $\mathrm{OB}$ associations that act collectively to build superbubbles.

Modeling this situation is approached at a variety of levels, e.g. Processes, Events, Local Region, and Global. At intermediate levels, one assumes that the processes at lower levels can be included in subgrid rules, while those at higher levels are embodied in initial or boundary conditions. One models the remnants of supernova explosions, for example, assuming an adequate understanding of the atomic and radiation processes, magnetic fields, thermal conduction, dust destruction and cosmic ray acceleration by shocks, etc., and a sufficient description of the surrounding medium. The results and their regime of applicability

(c) 2018 Kluwer Academic Publishers. Printed in the Netherlands. 
depend on the way in which all these lower and higher level aspects are approximated. The validity tends to increase with the degree to which the models are compared in intricate detail with the suite of observational data. With care, some parts may turn out to be right.

The assumptions we make are often those of convention; occasionally this may lead us astray. In general, astrophysicists are not comfortable with thermal conduction, for example. Magnetic fields interfere with it. Turbulence alters its basic nature (Lazarian and Cho, this volume). It is easy to leave it out of models because so many people believe it is quenched, while the others offer no clear prescription for how to include it. And yet it is a dangerous thing to omit in models that include hot gas. In two systems that I helped model, the Local Bubble and the W44 supernova remnant, the apparently observed characteristics appeared only when thermal conduction was included within the hot gas at roughly half the Spitzer rate. I've heard that something similar is being found in modeling cooling flows in clusters.

It is not clear how robust this hint is, but it begs for consideration. If you model the ISM, and find large volumes of hot gas, then please, to prevent our wasting too many more years in ignorance, add thermal conduction (with a variable amplitude parameter and limited by saturation) and see what its effects are. If your resolution is high enough that the turbulent and wandering field is adequately represented, let conduction cross your magnetic fields, with a reduced coefficient, perhaps of order $5 \%$ of the parallel rate (or ask Randy Jokipii what he would do). My prejudice is that thermal conduction in high temperature gas somehow manages to occur, despite magnetic fields, but that it is unable to cross boundaries to denser regions and thermally evaporate them. Maybe your models will give us a better sense of why.

Modeling superbubbles has a long history, including an early fascination with bubbles breaking out of the gaseous disk to dump their hot interiors into the Galactic halo. When it was finally realized that the gaseous disk is very thick in low density and nonthermal components, the fascination went away. Even as large as they are, the bubbles are confined within the the disk, which they help inflate, consistent with $\mathrm{x}$-ray observations of other galaxies that find very few plumes of hot gas rising out of their disks. The fascination with breakout was predicated on misinformation at the larger scales.

But modeling superbubbles may have problems beyond finding a reasonable representation of the ambient medium. Have we got the process details of the interior right? Do the winds of the OB stars, augmented perhaps by entrained material from the molecular cloud birthplace, hit the shell and set up a reverse shock, applying the accumulated pressure to the bubble walls? Or is the material so dilute that there is 
no collisionless shock in the wind? Does the fluid description give us the wrong answer? (I ask because Sally Oey is trying to understand why so many wind bubbles appear smaller than they theoretically ought to be. See her contribution to this volume.) We have great theories, but they do not encompass the observed facts. (But, Don Osterbrock would often say something like, "Never let questionable observations interfere with your appreciation of a good theory." The corollary that most observations can be questioned is one of Sally's approaches.)

In short, there are areas of ISM research in which important details are too poorly known for us to be confident in the ways we include them in models at other levels. That said, I am enormously impressed by the incredibly detailed models that are starting to appear. This meeting was very good in presenting the remarkable ambition of the current enterprises.

\section{There Are Many Conceptions of the ISM, All Flawed.}

What pictures would you draw to illustrate what you think are the dominant features of the ISM? I ask because what you draw determines what you put in your computer model, or how you describe the significance of your observations.

There are several commonly held conceptions, at different scales. Let's begin with conceptions within the disk, near us. The ones I will describe are the trivial ones. People such as Miguel de Avillez, who make models of the medium based on their best guesses of the process behaviors might draw something much more complex. One might even appear on the cover of this book, as it did on the book of abstracts.

Local conceptions tend to diverge over the component of the medium occupying the greatest volume. The three favorite possibilities appear to be:

- A warm intercloud medium, density perhaps averaging about 0.2 $\mathrm{cm}^{-3}$ and temperature perhaps $7000 \mathrm{~K}$, in some places ionized and others not.

- A pervasive hot component with a temperature of roughly $10^{6} \mathrm{~K}$ and pressure $p / k \sim 10^{4} \mathrm{~cm}^{-3} \mathrm{~K}$.

- A tepid intercloud component with a temperature of $1-3 \times 10^{5}$ $\mathrm{K}$.

The first of these views derives from the legacy of Field, Goldsmith and Habing (1969). It is somewhat supported observationally by the 
fact that there are warm $\mathrm{HI}$ and diffuse HII components that might be able to fill most of the volume. McKee and Ostriker (1977) argued that supernovae would disrupt such a medium and that the picture was therefore untenable. Slavin and Cox (1993), however, located the three fallacies leading to that conclusion, showed that supernovae might not disrupt such a medium, and found that invisible old remnants within it could provide all of the observed high stage ions (e.g. OVI) found in absorption measurements through the disk. Spitzer (private communication) however, wondered how the warm intercloud medium might go about restoring its relative uniformity. That question aside, it remains a somewhat attractive possibility, even though the detailed reasons for why it might be about right are unclear.

The second possibility follows from a suggestion by Cox and Smith (1974), and one much earlier by Spitzer (1956). The soft x-ray background appearing to derive from a large region of million Kelvin gas surrounding the Solar location led Cox and Smith to ask whether such gas might be common. I have abandoned this possibility because I think there is no observational evidence to support it, as well as no clear reason why it might be the preferred situation. I could be wrong; my distaste hinges largely on the expectation that it would have shown up more convincingly in soft x-rays. If, on the other hand, it were a few times hotter in most places, at a correspondingly lower density (to not have an unacceptably large pressure), its x-ray emission would be negligible and the scenario observationally allowed. (I like to refer to this as the Trans-Mega-Kelvin Regime.)

McKee and Ostriker (1977) suggested that there would likely be a thermal runaway in the hot component of Cox and Smith, unless one invoked a thermostatic mechanism such as evaporation of clouds to add mass. In this way, they evolved to the third scenario above. Their medium is heated to very high temperatures in local regions by supernovae, evaporates clouds, lowers its temperature as it expands and gains mass, and finally is able to radiate the supernova power when the temperature has dropped to a few hundred thousand Kelvins. The observed OVI was said to be in the thermally evaporating boundaries of the clouds, with the right average column density. The fact that far more OVI would be present in the tepid ambient medium, more than is observed by a large factor, was overlooked. For years I was convinced that the MO model was inconsistent with observation, but could not figure out how it failed. As remarked earlier, Jon Slavin and I finally found sufficient reason. It was a great example of observations insisting that the theory was lacking.

There is a fourth possibility that I try to remember to mention, thanks to a casual remark by Priscilla Frisch, in 1986. Perhaps most of 
the space is empty! This appears to be the impression of observers who study galactic sightlines and find components that fill only 20 or $30 \%$ of the total line of sight. Their copout, if they mention it at all, is that the rest of the sightline must be occupied by hot gas as advocated by ... someone else.

I'll try to make empty space sound not so crazy. It isn't that there is nothing in the empty regions. The magnetic field and cosmic rays are still there; the field lines just have very little mass on them. It's like an extreme version of the Trans-Mega-Kelvin regime. There is no observational reason to reject this conception; we just have no theoretical framework in which it is plausible. How would such empty regions come into existence (McKee and Ostriker's thermal runaway perhaps)? How do they evolve? Do they exit the Galaxy because of their buoyancy, or evolve out of existence via material leaking (or being driven) into them? Sound crazy still? Well, the last I heard, the outer shock of the Crab Nebula had not been found. The explosion appears to be expanding into a vacuum.

With regard to the more complicated pictures being drawn by large scale modelers, I can only agree with Joel Bregman that until they are able to tell us how much OVI is in their descriptions, we can't look at them with a sufficiently appreciative eye. I'll wager they get at least an order of magnitude too much! It is a powerful applicability check, hence the subtitle of this paper.

Then there are the vertical and the global conceptions. If the galaxy were truly filled with hot gas (not tied to a magnetic field anchored by the weight of the cold gas), such gas would extend far above the disk of the Galaxy, filling out a scale height at least. If many superbubbles grow so large that they break out of the disk, the hot gas from their interiors might plausibly fill the halo, the density rising until the hot gas is able to radiate the supernova power. Or, if the escaping gas were fairly dense, it might generate a fountain, cooling before it gets very far, condensing into clouds that eventually rain back. So, we have two scenarios, a hot quiescent halo, and a fountain. But we know from observations that the halos of galaxies do not radiate even a small fraction of the total supernova power, not at high temperatures. Both scenarios failed badly, to the great surprise of many of us who were expecting otherwise when x-ray data first became available.

What's left then? Well, cosmic rays escape the Galaxy. The phenomenon is frequently described as a wind. Perhaps the Galaxy has a thermal wind too, arising from the disk, high enough in temperature (and therefore low enough in density) that its x-ray emission is negligible (the Trans-Mega-Kelvin regime again). I believe that such a wind cannot be carrying away thermal energy faster than the cosmic ray loss 
rate, which is roughly $10 \%$ of the total supernova power. The cosmic rays seem to diffuse out of the disk, forming their "wind" rather high off the plane. If hot gas flows out rather unimpededly, it ought to take cosmic rays with it, in which case the two would leave the Galaxy in proportion to their relative energy densities in the hot component in the disk, a ratio of order unity in this scenario.

An entirely different possibility is that except for cosmic rays leaving the Galaxy, all other disturbances are trapped in the disk by the inelasticity of the great interstellar feather pillow. Superbubbles are the largest disturbances, but few reach such great size they are other than trapped volumes of hot gas that eventually dissipate their energies radiatively. This has been my favorite view for some time. It does not mean that there is no hot gas above the plane of the Galaxy; there are superbubbles that expand several hundred parsecs. We see several, and hot gas within them. There are also supernovae that occur at high $|z|$. These may be an important contributor to the thick layer in which high stage ions are found, as explored by Robin Shelton (1998). It just means that violent disturbances are generally locally confined, and dissipated.

What picture would you draw? Mine has all the usual components. There is a thick layer of low density warm intercloud gas, reaching up a kiloparsec or two, with here and there a confined supernova remnant within it. Quite a bit of it is ionized and visible in $\mathrm{H}$ alpha, the Reynolds Layer. It is pervaded by a largely random magnetic field and cosmic rays. Within it, dense cold clouds lie close to the midplane creating $\mathrm{OB}$ associations, the latter forming superbubbles that expand, but not to the extent that they break out of the disk. To be more precise, I think they help inflate the disk, rather than breaking out of it, except in the extreme case of starburst galaxies. Then there is the emptiness, emptiness that fills the ancient and dying carcasses of once grand superbubbles, à la the models of Ferrière (1998), and maybe even the emptiness of Frisch or the Trans-Mega-Kelvin regime. And finally, the whole thing is circulating around the Galaxy, now and then provoked into unusual activity by its encounter with a spiral arm. This spiral arm encounter is often described as a shock, but, Martos and Cox (1998) showed that it should have some elements of both a shock and a hydraulic jump, or bore. That work has been extended to three dimensions and a significant fraction of the Galaxy by Gómez and Cox (2002). A brief report appears in the poster contributions to this volume. It's exciting and an excellent example of a large-scale model that has to make very simple approximations to the character of the medium. There could also be a wind from the Galactic Bulge, providing a very interesting outer boundary condition on the ISM of the disk. 


\section{Local Matters Matter.}

This is about building a house of cards. It concerns the structure of the ISM in the vicinity of the Sun, starting out about 100 pc away and working in to about $5 \mathrm{AU}$. It is a progress report on years of work, and the fearful tests that lie ahead. Parts of it are discussed at length in Smith and Cox (2001) and Cox and Helenius (2003). Other authors have taken different routes with the observational data; references and discussions of their views appear in the papers above. But I am here offering an example of a modeling effort that has gone out on a limb, not surveying the whole realm of possibilities.

The Local Cavity is a large region of exceptionally low density surrounding the Solar location. Within it, there appears to be a sub-region of million Kelvin gas, the Hot Local Bubble, providing much of the soft $\mathrm{x}$-ray background. To get the observed $\mathrm{x}$-ray surface brightness, given the scale of order $100 \mathrm{pc}$, the required thermal pressure is $p / k \sim 2 \times 10^{4}$ $\mathrm{cm}^{-3} \mathrm{~K}$. This is just what would be expected for such a bubble quasistatically confined by the surrounding ISM, where the pressure of the latter equals its weight per unit area. Within that hot gas, there are wisps of higher density cooler material, the Local Fluff. The densities in the Local Fluff are of order $0.2 \mathrm{~cm}^{-3}$, the temperatures about $7000 \mathrm{~K}$, characteristic sizes of about 5 pc. One particular piece of this Fluff, the Local Cloud, surrounds the Solar System. The relative velocity between the Local Cloud and the Sun is about $26 \mathrm{~km} \mathrm{~s}^{-1}$. Thus, in the frame of the Sun, the material of the Local Cloud (the LISM, and VLISM), constitutes an interstellar wind blowing past the Solar System.

The Solar wind interacts with this interstellar wind, its momentum creating a large cavity around which the flow of interstellar ions and magnetic field is diverted. To first order, the neutral component of the Local Cloud does not notice this disturbance. The scale is sub-fluid for the neutrals, which tend to flow undisturbed into the heliosphere. (There is some charge exchange between the neutrals and diverted interstellar ions that has important consequences for the details.) Once they are deep into the Solar System, these neutrals suffer various fates. When one becomes ionized, it is immediately picked up by the Solar Wind magnetic field and joins the outflow; these are observed as "pickup ions" by interplanetary probes. On reaching the Solar Wind termination shock, some are accelerated to higher energies, diffuse back in toward the Earth, and are observed as the "anomalous cosmic rays." Radiation pressure from scattered Solar Lyman alpha approximately cancels gravity, so the hydrogen trajectories are straight. Solar radiation ionizes most of it before it reaches $5 \mathrm{AU}$. The distribution of interstellar hydrogen in the inner Solar System is therefore roughly uniform in the 
upstream direction, outward of $5 \mathrm{AU}$, and absent at smaller radii and in much of the downstream direction. It is observed via its backscattering of the Solar Lyman alpha. Helium suffers less radiation pressure and ionization, and is gravitationally focused in the downstream direction. This is observed via resonant backscattering of $304 \AA$ Solar radiation.

Smith and Cox (2001) explored the possibility that the Local Bubble could have been created by an accidental sequence of supernovae in a warm intercloud medium as described in Section 2. They found that a sequence of three supernovae over the course of several million years, the second and third each occurring within the region previously evacuated, could create a bubble of radius about $100 \mathrm{pc}$, and leave it with the right density and temperature so that its radiation would resemble the soft x-ray background. Considering that the Local Cavity is much larger in some directions, and potentially much older, a generalization is that something created the large cavity, and a supernova reheated a portion of it roughly three million years ago to leave us surrounded by the Hot Local Bubble. Evidence for the nearby occurrence of a supernova about that long ago is discussed in their paper and further in Cox and Helenius(2003).

But why would the Local Fluff be sitting in the middle of such a remnant? A possible answer was provided by Cox and Helenius (2003) who examined the evolution of a magnetic flux tube pulled from the wall of the bubble by its magnetic tension, starting about three million years ago, after it had been swept to the wall by the supernova. As the flux tube springs from the wall, it is initially accelerated very rapidly; but soon drag through the hot gas becomes important, and it gradually decelerates. Material within the tube starts out very diffuse, part of the inner wall of the bubble, but the initial acceleration due to tension is largely radial. As the tube itself slows, the material within it flows toward its center, concentrating into a low-density cloud, or collection of interacting clouds, depending on the complexity of the initial conditions. By the time the tube reaches the bubble middle, the velocity distribution of the material within resembles that observed in the Local Fluff, in magnitude, and in the generally converging flow pattern. Its density and ionization state are also appropriate. The details need more study, but the scenario creates something very much like the observed Local Fluff.

Now we turn to the problems. Understanding of the soft x-ray background is not complete. High spectral resolution will eventually give us a much better idea of what we are looking at. For the present, there is at least one disturbing possibility. The sky maps of the SXRB from ROSAT were made from data that had contamination that was not understood at the time, the "Long Term Enhancements." In map- 
ping, periods of time during which this contamination was large were omitted. The maps thus present the lowest signal detected from each direction. Their spatial structure correlates very well with maps from rockets, and Galactic structure, so it is believed that the process was successful in removing the contamination. But the resulting $1 / 4$ th $\mathrm{keV}$ surface brightness is pretty uniform in the Galactic Plane, getting brighter at higher latitudes. The worry is that the midplane intensity could be just the DC level of the contamination. If so, we would lose the Local Bubble as we think we know it, replacing it with smaller bubbles at positive and negative higher latitudes. After the observations of x-rays from comets, and their interpretation as arising from charge exchange recombination of highly ionized elements in the Solar Wind, we finally had a mechanism for generating x-rays within the Solar System. It now seems likely that the Long Term Enhancements arise from the same mechanism, with the charge exchange occurring on the atoms of the Earth's exosphere. There should be also be a significant steady contribution from the Solar Wind ions charge exchanging on the interstellar neutrals penetrating the Solar System (e.g. Cox, 1998, Cravens et al., 2001). The optimistic view from the estimates so far is that the level of the overall contamination is modest, at $30 \%$ or so, and that our picture will remain largely undisturbed. We'll see.

The other major problem is the distance to the Solar Wind Termination Shock. I told you what the pressure in the Local Bubble had to be to produce the observed x-rays, and that it is appropriate for a bubble in equilibrium with the surrounding ISM. I also gave you data from which you can estimate the thermal pressure in the Local Fluff. The latter is less than $20 \%$ of the former. In the model of Cox and Helenius, the magnetic pressure of the flux tube is significantly higher than the thermal pressure of the gas within it. The field strength is several microGauss, to balance the thermal pressure of the surrounding hot bubble. Forget the model. In order for the Local Fluff to be in pressure equilibrium with the surroundings, such a field is required. But modelers of the interaction of the Solar Wind with the Local Cloud will tell you that if the magnetic pressure in the Local Cloud were that high, the termination shock would have been pushed inward to the point that Voyager 1 would already have encountered it, which it has not! There are only a few ways out of this difficulty. One is that making such models is difficult (as in the theme of Section 1) and details may have been overlooked. Astronomically insignificant things like a measly factor of two are critical. Another is that the Cox and Helenius model makes a specific prediction for the direction of the magnetic field, from the directionality of the local distribution of Fluff, and that direction 
is roughly parallel to the relative wind flow. This is a case that has not been examined and offers a radically different structure.

A third alternative is the scary one, involving both of the above problems. The field is actually much weaker. The Local Bubble does not exist. The local interstellar total pressure is very significantly lower than the average expected from the weight of the interstellar medium. The whole house of cards collapses. We'll see.

One last thing: If you model the ISM, don't forget to tell us how much OVI you get. Send me a telegram when you no longer find more than a factor of two too much!

This work was supported in part by NASA grant NAG5-8417 to the University of Wisconsin-Madison. It has benefited from critical readings by Gilberto Gómez, Bob Benjamin, Sally Oey, Maarit Korpi, and Dieter Breitschwerdt. I apologize to the many observers whose work made the above discussion possible yet who were not mentioned by name or referenced. To others, please do not suppose that I have told you the truth about observations. Go to the sources.

\section{References}

Cox, D.P., 1996a, in Astrophysics in the Extreme Ultraviolet, Kluwer, eds. S. Bowyer and R. F. Malina, p. 247

Cox, D.P., 1996b, in proceedings of the Institute d'Astrophysique meeting On The Interplay Between Massive Star Formation, the ISM, and Galaxy Evolution, in Paris, eds. Kunth et al., Editions Frontieres, p. 233

Cox, D.P., 1998, In IAU Colloq. 166, The Local Bubble and Beyond, ed. D. Breitschwerdt, M.J. Freyberg, \& J. Trumper (Berlin: Springer), 5

Cox, D.P. and Helenius, L. 2003, ApJ, 583, 205

Cox, D.P. and Smith, B.W. 1974, ApJLett, 189, L105

Cravens, T.E., Robertson, I.P., and Snowden, S.I. 2001, JGR, 106, 24883

Ferrière, K. 1998, ApJ, 503, 700

Field, G.B., Goldsmith, D.W., and Habing, H.J. 1969, ApJL, 155, L149

Gómez, G.C. and Cox, D.P. 2002, ApJ, 580, 235

Martos, M.A. and Cox, D.P. 1998, ApJ, 509, 703

McKee, C.F. and Ostriker, J.P. 1977, ApJ, 218, 148

Shelton, R.L. 1998, ApJ, 594, 785

Slavin, J.D. and Cox, D.P. 1993, ApJ, 417, 187

Smith, R.K. and Cox, D.P. 2001, ApJS, 134, 283

Spitzer, L., Jr. 1956, ApJ, 124, 20 